\title{
Breast cancer stage at diagnosis and area-based socioeconomic status: a multicenter 10-year retrospective clinical epidemiological study in China
}

Qiong Wang ${ }^{1,2}$, Jing $\mathrm{Li}^{2}$, Shan Zheng ${ }^{3}$, Jia-Yuan $\mathrm{Li}^{i^{*}}$, Yi Pang ${ }^{1}$, Rong Huang ${ }^{1,2}$, Bao-Ning Zhang ${ }^{4}$, Bin Zhang ${ }^{4,5}$,

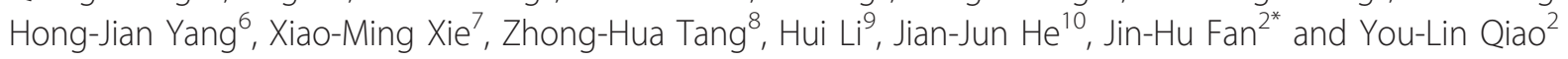

\begin{abstract}
Background: Although socioeconomic status (SES) has been focused on as a key determinant of cancer stage at diagosis in western countries, there has been no systemic study on the relationship of SES and breast cancer stage at diagnosis in China.

Methods: The medical charts of 4,211 eligible breast cancer patients from 7 areas across China who were diagnosed between 1999 and 2008 were reviewed. Four area-based socioeconomic indicators were used to calculate area-based SES by cluster analysis. The associations between area-based SES and stage at diagnosis were analyzed by trend chi-square tests. Binary logistic regression was performed to estimate odds ratios for individual demographic characteristics' effects on cancer stages, stratified by area-based SES.

Results: The individual demographic and pathologic characteristics of breast cancer cases were significantly different among the seven areas studied. More breast cancer cases in low SES areas (25.5\%) were diagnosed later (stages III \& IV) than those in high $(20.4 \%)$ or highest $(14.8 \%)$ SES areas $\left(\chi^{2}\right.$ for trend $\left.=80.79, P<0.001\right)$. When areabased SES is controlled for, in high SES areas, cases with less education were more likely to be diagnosed at later stages compared with more educated cases. In low SES areas, working women appeared to be diagnosed at earlier breast cancer stages than were homemakers (OR: 0.18-0.26).
\end{abstract}

Conclusions: In China, women in low SES areas are more likely to be diagnosed at later breast cancer stages than those in high SES areas.

Keywords: Breast cancer, Stage at diagnosis, Area-based socioeconomic status, Nation-wide, Multi-center, Retrospective study

\section{Background}

Breast cancer is by far the most common cancer among women both in developed and developing regions, with an estimated 1.38 million new cancer cases diagnosed worldwide in 2008 (23\% of all cancers) [1]. In recent years, both incidence of and mortality from breast

\footnotetext{
* Correspondence: lijiayuan73@163.com; fjhjjj@sohu.com

'Department of Epidemiology, West China School of Public Health, Sichuan University, 16 Ren Min Nan Lu, Chengdu, Sichuan 610041, China

${ }^{2}$ Department of Cancer Epidemiology, Cancer Institute \& Hospital, Chinese Academy of Medical Sciences \& Peking Union Medical College, 17 South

Panjiayuan Lane, Beijing 100021, China

Full list of author information is available at the end of the article
}

cancer have declined in the United States; between 1999 and 2006 , incidence rates decreased by $2.0 \%$ per year, and mortality decreased by $1.9 \%$ annually between 1998 and 2006 [2]. However, the incidence of breast cancer is steadily rising in developing countries [3-6]. In China, the incidence of breast cancer rose from 126,227 cases in 2002 [7] to over 169,000 in 2008 [1].

Preventive screening for breast cancer can increase the number of individuals diagnosed at an early stages and reduce mortality. In China, the main methods of breast cancer screening are clinical breast examination (CBE), mammography, high-frequency ultrasound, and 
magnetic resonance imaging (MRI). However, due to the lack of large population-based screening evaluations in China, there is no consistent evidence indicating which screening method or combination of methods is best for the country. To date, nationwide breast cancer screening has never been implemented routinely in China, and in places where routine breast cancer screening exists, its usage varies with area-based socioeconomic status (SES) $[8,9]$. Individuals living in poorer areas are less likely to seek cancer screening compared with individuals living in wealthier areas [10]. Studies over the past several decades have indicated that individuals living in less developed areas often had worse general health compared with individuals living in relatively developed areas [11-17]. The majority of studies investigating associations between SES and breast cancer stage at diagnosis have also documented socioeconomic and geographic disparities, with higher incidence of late stage breast cancer in lower income areas [18-24]. Regional disparities in SES are significant in China, where economic development in eastern cities generally started earlier and has been faster than that in the country's interior and in rural areas [25].

Breast cancer survival rates decline with delayed diagnosis. The Surveillance Epidemiology and End Results (SEER) showed that $98.3 \%$ of women treated for earlystage breast disease survive five or more years, while only $83.5 \%$ of women diagnosed with regional breast cancer and $23.3 \%$ of those with distant breast cancer survive beyond five years of the initial diagnosis [2]. Thus, as improvement in breast cancer prognosis would have a significant impact on countries' health budgets, and SES disparities result in different breast cancer outcomes, strategies are urgently needed that target higher-risk areas and resonate with higher-risk population subgroups. Breast cancer prevention and control has been prioritized by China's Ministry of Health, but so far, there has been no systemic study of the relationship between SES and breast cancer stage in the country. In this study, based on the Nationwide Multicenter 10-year (1999-2008) Retrospective Clinical Epidemiological Study of Breast Cancer in China, directed by the Cancer Hospital/Institute, Chinese Academy of Medical Sciences (CICAMS), we explored the effects of both area level factors, namely SES, and individual demographic characteristics on breast cancer stage at diagnosis.

\section{Methods}

\section{Hospital selection, case sampling, and data collection}

Hospital selection, case sampling, and data collection methods have been previously described in detail [26]. Briefly, all of China was stratified into seven geographic areas (north, northeast, central, south, east, northwest, and southwest). Then, purposive sampling was used to choose one tertiary public cancer hospital in each region with the following characteristics. Firstly, participant hospitals were the leading public cancer hospitals and regional referral centers providing pathology diagnosis, surgery, radiotherapy, medical oncology, and routine follow-up care for patients with breast cancer. Secondly, their patient source had to include the entire study region. Finally, since patients tend to seek better medical service in big cities, the hospitals were each located in a major city. The participant hospitals were the Cancer Institute \& Hospital, Chinese Academy of Medical Sciences (in Beijing city, north China), Liaoning Cancer Hospital (in Liaoning Province, northeast China), Second Xiangya Hospital, Central South University (in Hunan Province, central China), Sun Yat-Sen University Cancer Center (in Guangdong Province, south China), Zhejiang Cancer Hospital (in Zhejiang Province, east China), First Affiliated Hospital of Xi'an Jiaotong University (in Shannxi Province, northwest China), and Sichuan Cancer Hospital (in Sichuan Province, southwest China).

The selected hospitals provided us with the medical records of breast cancer patients diagnosed between 1999 and 2008. For each year in each hospital, one month was randomly selected, and all inpatient cases for that month were reviewed (January and February were excluded from the random selection to eliminate any confounding effects of China's largest annual holiday). The hospital records were reviewed in each local hospital by local clerks who had been trained systematically in Beijing. Then, standard case report forms (CRF) designed by CICAMS [26] was used to extract the primary medical reports of every qualified patient, including general information, risk factors, diagnostic imaging tests, therapy models, and pathologic characteristics. The reliability and validity of CRF had been assessed by proceeding a pilot study and CRF was regarded as reliable and valid. After collection, the raw variables were coded and sorted into different categories for analysis. Cancer stage at diagnosis was categorized into six groups by archiaters referring to pathologic reports and using the American Joint Committee on Cancer (AJCC) TNM System (0, I, II, III, IV, and unknown stage or not applicable). The cases admitted between 1999 and 2002 were staged using the fifth version (revised in 1997) [27], and the cases from 2003-2008 were staged with the sixth version (revised in 2002) [28]. If individuals were diagnosed with more than one primary breast tumor at the same time, we included only the record of the tumor at the most advanced stage. We excluded tumors with an unknown or not applicable stage, and grouped the breast cancer stages of the other cases into two categories: "early" (stages 0 \& I) or "non-early" (stages II \& III \& IV). 
The study protocol was approved by the Cancer Foundation of China Institutional Review Board and data were stripped of all personal identifiers, per the board's approved procedures.

\section{Area-based socioeconomic indicators}

Area-based SES measures derived from census data have been shown to be related to various health outcomes independent of individual SES [11,12,22,29,30]. Because there is a lack of accepted SES measures in China, we referred to the socioeconomic measures of "percent of the population living below poverty line" and "percent of the population $\geq 25$ years of age without a high school diploma" in the United States [31] to devise our own socioeconomic indicators (SEIs) reflecting the economic and education status of the seven areas. These are "GDP per capita," "percentage of health-service expenditure in the regional/provincial public affairs general budget (HSE percentage)," "ratio of urban to rural population (PU/PR ratio)," and "percentage of illiteracy in females aged 15 and over (FPI percentage)." The SEIs of each district or province with a selected hospital were obtained from annual reports issued by the National Bureau of Statistics of the People's Republic of China from 1999 to 2008, except that data on the PU/PR ratio was available only from 2005 to 2008 . These SEIs were used to represent the SES of each area.

\section{Data Analysis}

Descriptive statistics were used to summarize the demographic and pathologic characteristics of the study population. The mean and standard deviation of quantitative variables were calculated, and the differences among the seven areas were analyzed with one-way ANOVA. Differences in distribution of variables were examined using Mantel-Haenszel chi-square tests or Fisher's exact tests.

The single SEI values among the seven areas were compared using one-way ANOVA followed by the Student-Newman-Keuls (SNK) test, a post hoc tests that measures significance among multiple groups. Those areas between which there were no statistically significant differences were combined, forming ordinal categorical levels of SEI status. Trend chi-square tests were used to examine associations between stage at diagnosis and the SEIs status. Additionally, area-based SES was calculated by cluster analysis, an exploratory data analysis tool which sorts objects into groups such that the degree of association between two objects is maximal if they belong to the same group and minimal otherwise. During cluster analysis, the k-means clustering was applied with $\mathrm{k}=3$, so that the seven regions were classified into three levels based on four SEIs. Binary logistic regression was performed stratified by area-based SES to further explore the association between individual demographic characteristics and breast cancer stage at diagnosis (non-early stage vs. early stage) in SES subgroups. SPSS statistical software version 17.0 was used to analyze the data. Statistical significance was assessed by two-tailed tests with an $\alpha$ level of 0.05 .

\section{Results}

SEls, individual demographic and pathologic characteristics by area

A total of 4,211 eligible cases were included in our study. In general, the differences in SEIs among the seven areas were significant $(P<0.001)$ (Table 1$)$. During the period studied, the mean of GDP per capita across the seven areas was 13,048 $\pm 5,567$ yuan per person, ranging from $8,050 \pm 3,599$ in the southwest to $36,146 \pm 17,217$ in northern China. More than $70 \%$ of breast cancer patients were 40-69 years old when diagnosed. Cases in the north (42.1\%), northeast (32.8\%), and south $(29.8 \%)$ were more likely to be overweight at diagnosis $\left(B M I \geq 25.00 \mathrm{~kg} / \mathrm{m}^{2}\right)$ than were those in other areas $(3.9 \%-18.8 \%)$. Women in eastern, northwestern, and southwestern China were mainly occupied in manual work $(80.4 \%, 54.0 \%$, and $62.7 \%$, respectively), while more women were homemakers in central China $(13.2 \%)$ than in other areas (average across all areas = $4.0 \%)$. Information on patients' education levels was generally unavailable in north, northeast, and southwest China. Among the other four areas, we found that women in central China had higher education levels, with $27.5 \%$ receiving higher education (university and above). The vast majority of cases in all seven areas were married. All the above demographic characteristics of breast cancer cases were significantly different among the seven areas (Table $2, P<0.001$ ).

Invasive ductal carcinoma was the dominant pathologic subtype, ranging from $70.1 \%$ of cases in the northeast to $92.4 \%$ in central China. Among 3555 cases that were tested for estrogen receptor (ER) and progesterone receptor (PR), 3534 had test results included in their records. About half of these (47.6\%) were ER + and PR+, and $32.0 \%$ were ER- and PR-. In northwest China, only $282(58.4 \%)$ cases were tested for ER/PR and $38.7 \%$ of them were $\mathrm{ER}+$ and $\mathrm{PR}+$; both of these rates were far lower than in the other areas. Human epidermal growth factor receptor 2 (Her-2) information was available for 2849 patients, the majority of whom $(2113,65.0 \%)$ were Her-2 negative. The north had a high testing rate $(609 / 641,95.0 \%)$ and the highest positive rate $(286 / 609,47.0 \%)$ for Her-2. All of the pathologic characteristics of breast cancer cases were significantly different across the seven areas (Table 3, $P<0.001)$. 
Table 1 SEls of seven regions

\begin{tabular}{|c|c|c|c|c|c|c|c|c|c|c|}
\hline $\begin{array}{l}\text { Variable by } \\
\text { level }\end{array}$ & $\begin{array}{c}\text { Total } \\
\text { (Range) }\end{array}$ & $\begin{array}{l}\text { Beijing } \\
\text { (North) }\end{array}$ & $\begin{array}{l}\text { Liaoning } \\
\text { (Northeast) }\end{array}$ & $\begin{array}{l}\text { Hunan } \\
\text { (Central) }\end{array}$ & $\begin{array}{l}\text { Guangdong } \\
\text { (South) }\end{array}$ & $\begin{array}{l}\text { Zhejiang } \\
\text { (East) }\end{array}$ & $\begin{array}{c}\text { Shannxi } \\
\text { (Northwest) }\end{array}$ & $\begin{array}{c}\text { Sichuan } \\
\text { (Southwest) }\end{array}$ & $\begin{array}{c}F(P- \\
\text { value })\end{array}$ & $\begin{array}{c}\text { SNK* } \\
\text { (No. of } \\
\text { subgroups) }\end{array}$ \\
\hline \multicolumn{11}{|l|}{ SEI } \\
\hline $\begin{array}{l}\text { GDP per } \\
\text { capita }^{(1)}\end{array}$ & $\begin{array}{c}13048 \pm \\
5567 \\
(8050- \\
36146)\end{array}$ & $\begin{array}{c}36146 \pm \\
17217^{a}\end{array}$ & $\begin{array}{l}17200 \pm \\
6762^{\mathrm{b}}\end{array}$ & $\begin{array}{l}9223 \pm \\
4019^{c}\end{array}$ & $\begin{array}{l}21292 \pm \\
9165^{\mathrm{b}}\end{array}$ & $\begin{array}{l}23832 \pm \\
10331^{\mathrm{b}}\end{array}$ & $\begin{array}{l}8840 \pm \\
4615^{c}\end{array}$ & $\begin{array}{l}8050 \pm \\
3599^{c}\end{array}$ & $\begin{array}{c}12.69 \\
(< \\
0.001)\end{array}$ & 3 \\
\hline $\begin{array}{l}\text { HSE } \\
\text { percentage }^{(2)}\end{array}$ & $\begin{array}{c}4.47 \pm \\
0.43 \\
(3.18-6.51)\end{array}$ & $6.51 \pm 0.51^{a}$ & $3.18 \pm 0.41^{c}$ & $\begin{array}{l}3.41 \pm \\
0.74^{c}\end{array}$ & $4.27 \pm 0.45^{b}$ & $5.71 \pm 0.71^{a}$ & $3.87 \pm 0.75^{c}$ & $4.56 \pm 0.66^{b}$ & $\begin{array}{c}38.69 \\
(< \\
0.001)\end{array}$ & 3 \\
\hline PU/PR ratio ${ }^{(3)}$ & $\begin{array}{c}0.80 \pm \\
0.04 \\
(0.54-5.39)\end{array}$ & $5.39 \pm 0.22^{\mathrm{a}}$ & $1.45 \pm 0.03^{c}$ & $0.66 \pm$ & $1.67 \pm 0.09^{b}$ & $1.32 \pm 0.04^{c}$ & $0.66 \pm 0.06^{d}$ & $0.54 \pm 0.05^{d}$ & $\begin{array}{c}1210 \\
(< \\
0.001)\end{array}$ & 4 \\
\hline $\begin{array}{l}\text { FPI } \\
\text { percentage }^{(4)}\end{array}$ & $\begin{array}{c}15.17 \pm \\
2.98 \\
(7.20- \\
17.74)\end{array}$ & $7.21 \pm 1.63^{a}$ & $7.20 \pm 1.60^{a}$ & $\begin{array}{l}11.31 \pm \\
2.60^{\mathrm{b}}\end{array}$ & $9.89 \pm 2.57^{b}$ & $\begin{array}{l}17.30 \pm \\
3.07^{c}\end{array}$ & $\begin{array}{l}15.86 \pm \\
4.30^{c}\end{array}$ & $\begin{array}{c}17.74 \pm \\
3.31^{c}\end{array}$ & $\begin{array}{c}23.02 \\
(< \\
0.001)\end{array}$ & 3 \\
\hline
\end{tabular}

\footnotetext{
*: The single SEI values among the seven areas were compared using one-way ANOVA followed by the Student-Newman-Keuls (SNK) test, a post hoc test for significant differences among multiple groups. The areas between which there were no statistically significant differences were combined to form subgroups. $a, b, c$, and $d$ represent different subgroups for each variable; if two or more regions are followed by the same letter, there is no significant difference between them.

${ }^{(1)}$ : yuan per person per year (mean \pm SD, 1999-2008)

${ }^{(2)}$ : percentage of health-services expenditure in the regional/provincial public affairs general budget (\%) (mean \pm SD, 1999-2008)

(3): ratio of urban to rural population (mean \pm SD, 2005-2008) (data is available in the National Bureau of Statistics of the People's Republic of China only for 2005 to 2008)

(4): percentage of illiteracy among women aged 15 and over (\%) (mean \pm SD, 1999-2008)
}

SEls, area-based SES, and breast cancer stage at diagnosis For the analysis of factors affecting breast cancer stage at diagnosis, we excluded 717 cases with an unknown or not applicable stage, so that the final number of cases in the following analyses was 3494.

We found an inverse relationship between breast cancer stage and both GDP per capita and ratio of urban to rural population, while percentage of illiteracy in females aged 15 and over was positively related to breast cancer stage $(P$ for trend $<0.001)$ (Table 4$)$. However, there was no significant trend correlation between percentage of health-service expenditure in the regional/provincial public affairs general budget and breast cancer stage $\left(\chi^{2}\right.$ for trend $=3.307, P=0.081$ ). The area-based SES based on the four SEIs was negatively correlated with breast cancer stage: $14.8 \%(95 / 641)$ of cases in the highest SES areas were diagnosed at stages III or IV, while $20.4 \%$ (417/2024) of cases in high SES areas and 25.5\% (389/ $1528)$ in low SES areas were diagnosed at these late stages $\left(\chi^{2}\right.$ for trend $\left.=80.79, P<0.001\right)$. Patients in Beijing and Guangzhou, where routine breast cancer screening has been implemented for several years [32,33], were more likely to be diagnosed at stages 0 or I $(27.9 \%$ and $17.5 \%)$ than were patients in other areas (Table 3 ).

\section{Association between individual demographic characteristics and breast cancer stage at diagnosis stratified by area-based SES}

With the exception of percentage of health-service expenditure in the regional/provincial public affairs general budget, each of the SEIs was associated with breast cancer stage (Table 4). To further explore the association between individual demographic characteristics and breast cancer stage at diagnosis, we used binary logistic regression and implemented stratified analysis by area-based SES (Table 5). In north China, the only area categorized as highest SES, education information was unavailable for $50.3 \%$ of the cases, so we were unable to analyze the effect of individual demographic characteristics in this subgroup. In high SES areas, education appeared to influence the cancer stage at diagnosis. Compared with the patients who had attended university, those only attended middle and/or high school had about a three-fold higher risk of being diagnosed at a late stage. In low SES areas, working women (manual, white-collar, or other) were less likely to be diagnosed at late breast cancer stages than were homemakers (OR: 0.18-0.66). Other demographic characteristics, including age at diagnosis and marital status, were not associated with breast cancer stage at diagnosis.

\section{Discussion}

Increasing social inequalities in health in China, coupled with growing inequalities in income and wealth, have focused attention on social class as a key determinant of population health. Our study focused on breast cancer stage at diagnosis as an indicator of health care access and quality, and we found a significant relationship between cancer stage and area-based SES. To our 
Table 2 Individual demographic characteristics by region

\begin{tabular}{|c|c|c|c|c|c|c|c|c|c|c|}
\hline & Total & $\begin{array}{l}\text { Beijing } \\
\text { (North) }\end{array}$ & $\begin{array}{l}\text { Liaoning } \\
\text { (Northeast) }\end{array}$ & $\begin{array}{l}\text { Hunan } \\
\text { (Central) }\end{array}$ & $\begin{array}{l}\text { Guangdong } \\
\text { (South) }\end{array}$ & $\begin{array}{c}\text { Zhejiang } \\
\text { (East) }\end{array}$ & $\begin{array}{c}\text { Shannxi } \\
\text { (Northwest) }\end{array}$ & $\begin{array}{c}\text { Sichuan } \\
\text { (Southwest) }\end{array}$ & $\begin{array}{l}\text { Statistical } \\
\text { value }\end{array}$ & $\begin{array}{c}P- \\
\text { value }\end{array}$ \\
\hline \multirow[t]{2}{*}{$\begin{array}{l}\text { Demographic } \\
\text { Characteristic }\end{array}$} & $\begin{array}{c}\text { Total } N= \\
4211\end{array}$ & $\begin{array}{c}\text { Total N }= \\
641\end{array}$ & $\begin{array}{c}\text { Total } N= \\
832\end{array}$ & $\begin{array}{c}\text { Total N }= \\
546\end{array}$ & $\begin{array}{c}\text { Total } N= \\
604\end{array}$ & $\begin{array}{l}\text { Total N }= \\
606\end{array}$ & $\begin{array}{c}\text { Total N }= \\
483\end{array}$ & $\begin{array}{c}\text { Total } N= \\
499\end{array}$ & & \\
\hline & N (\%) & N (\%) & N (\%) & N (\%) & N (\%) & N (\%) & N (\%) & N (\%) & & \\
\hline \multicolumn{11}{|l|}{$\begin{array}{l}\text { Age at diagnosis } \\
\text { (years) }\end{array}$} \\
\hline Mean \pm SD & $\begin{array}{c}48.68 \pm \\
10.47\end{array}$ & $\begin{array}{c}50.45 \pm \\
10.92\end{array}$ & $\begin{array}{c}48.88 \pm \\
9.95\end{array}$ & $\begin{array}{c}48.25 \pm \\
10.34\end{array}$ & $\begin{array}{c}47.82 \pm \\
10.94\end{array}$ & $\begin{array}{c}47.35 \pm \\
9.58\end{array}$ & $\begin{array}{c}50.10 \pm \\
11.01\end{array}$ & $\begin{array}{c}47.86 \pm \\
10.32\end{array}$ & $7.63^{*}$ & $\begin{array}{c}< \\
0.001\end{array}$ \\
\hline$\leq 40$ & $919(21.8)$ & $113(17.6)$ & $161(19.4)$ & $133(24.4)$ & $155(25.7)$ & $147(24.3)$ & 89 (18.4) & $121(24.2)$ & & \\
\hline $40-69$ & $3126(74.2)$ & 489 (76.3) & $645(77.5)$ & 387 (70.9) & $429(71.0)$ & $443(73.1)$ & $369(76.4)$ & $364(72.9)$ & & \\
\hline$\geq 70$ & $166(3.9)$ & $39(6.1)$ & $26(3.1)$ & $26(4.8)$ & $20(3.3)$ & $16(2.6)$ & $25(5.2)$ & $14(2.8)$ & & \\
\hline \multicolumn{11}{|l|}{$\begin{array}{l}\text { Body Mass Index } \\
\left(\mathrm{kg} / \mathrm{m}^{2}\right)^{(1)}\end{array}$} \\
\hline Mean \pm SD & $\begin{array}{c}23.35 \pm \\
2.89\end{array}$ & $\begin{array}{c}24.65 \pm \\
3.46\end{array}$ & $\begin{array}{l}24.01 \pm \\
3.08\end{array}$ & $\begin{array}{c}21.72 \pm \\
1.85\end{array}$ & $23.25 \pm 3.47$ & $\begin{array}{c}22.28 \pm \\
2.86\end{array}$ & $23.25 \pm 1.29$ & $23.25 \pm 1.82$ & $67.42^{*}$ & $\begin{array}{c}< \\
0.001\end{array}$ \\
\hline$\leq 24.99$ & $3287(78.1)$ & $371(57.9)$ & $559(67.2)$ & $520(95.2)$ & $422(70.2)$ & $492(81.2)$ & $464(96.1)$ & $460(92.2)$ & & \\
\hline$\geq 25.00$ & $921(21.9)$ & $270(42.1)$ & $273(32.8)$ & $26(4.8)$ & $180(29.8)$ & $114(18.8)$ & $19(3.9)$ & $39(7.8)$ & & \\
\hline \multicolumn{11}{|l|}{ Occupation } \\
\hline Homemaker & $173(4.0)$ & $17(2.7)$ & $2(0.2)$ & $72(13.2)$ & $39(6.5)$ & $11(1.8)$ & $14(2.9)$ & $18(3.6)$ & $6.52^{* *}$ & $\begin{array}{c}< \\
0.001\end{array}$ \\
\hline Manual worker & $1893(45.0)$ & $230(35.9)$ & $258(31.1)$ & 185 (33.9) & 159 (26.3) & 487 (80.4) & $261(54.0)$ & $313(62.7)$ & & \\
\hline $\begin{array}{l}\text { White-collar } \\
\text { worker }\end{array}$ & $1028(24.4)$ & $203(31.7)$ & $174(20.9)$ & $168(30.8)$ & $167(27.6)$ & $61(10.1)$ & $153(31.7)$ & $102(20.4)$ & & \\
\hline Other & $529(12.6)$ & 70 (10.9) & 99 (11.9) & $114(20.9)$ & $162(26.8)$ & $40(6.6)$ & $19(3.9)$ & $25(5.0)$ & & \\
\hline Unknown & $588(14.0)$ & $121(18.9)$ & 299 (35.9) & 7 (1.2) & 77 (12.8) & $7(1.1)$ & $36(7.5)$ & $41(8.2)$ & & \\
\hline \multicolumn{11}{|l|}{ Education } \\
\hline None & $186(4.4)$ & $5(0.8)$ & $1(0.1)$ & $0(0.0)$ & $41(6.8)$ & $124(20.5)$ & $14(2.9)$ & $1(0.2)$ & $4.63^{* * *}$ & $\begin{array}{c}< \\
0.001\end{array}$ \\
\hline Primary school & 462 (11.0) & $3(0.5)$ & $0(0.0)$ & 125 (22.9) & $116(19.2)$ & $173(28.5)$ & $39(8.1)$ & $6(1.2)$ & & \\
\hline Middle school & $606(14.4)$ & $0(0.00)$ & $0(0.0)$ & 147 (26.9) & $172(28.5)$ & $165(27.2)$ & $115(23.8)$ & $7(1.4)$ & & \\
\hline High school & $441(10.5)$ & $0(0.00)$ & $2(0.2)$ & $122(22.3)$ & $143(23.7)$ & $56(9.2)$ & $116(24.0)$ & $2(0.4)$ & & \\
\hline $\begin{array}{l}\text { University and } \\
\text { above }\end{array}$ & $396(9.4)$ & $4(0.6)$ & $23(2.8)$ & $150(27.5)$ & $112(18.5)$ & $29(4.8)$ & 76 (15.7) & $2(0.4)$ & & \\
\hline Unknown & $2120(50.3)$ & $629(98.1)$ & 806 (96.9) & $2(0.4)$ & $20(3.3)$ & $59(9.8)$ & $123(25.5)$ & 481 (96.4) & & \\
\hline \multicolumn{11}{|l|}{ Marital Status } \\
\hline Single & $51(1.2)$ & $6(0.9)$ & $9(1.1)$ & $4(0.7)$ & $17(2.8)$ & $5(0.8)$ & $3(0.6)$ & $7(1.4)$ & $56.43^{* *}$ & $\begin{array}{c}< \\
0.001\end{array}$ \\
\hline Married & 4090 (97.1) & $620(96.7)$ & $821(98.7)$ & $525(96.2)$ & $586(97.0)$ & $589(97.2)$ & $474(98.1)$ & 475 (95.2) & & \\
\hline $\begin{array}{l}\text { Widowed/ } \\
\text { Divorced }\end{array}$ & $52(1.2)$ & $12(1.9)$ & $0(0.0)$ & $17(3.1)$ & $0(0.0)$ & $7(1.2)$ & $5(1.0)$ & $11(2.2)$ & & \\
\hline Unknown & $18(0.5)$ & $3(0.5)$ & $2(0.2)$ & - & $1(0.2)$ & $5(0.8)$ & $1(0.2)$ & $6(1.2)$ & & \\
\hline
\end{tabular}

knowledge, ours is the first study to tie SES to health status within a developing country.

In this nationwide multicenter study of breast cancer in China, area-based SES was categorized into three levels: highest, high, and low. The Beijing district in north China was the only highest SES area, and also had the highest GDP per capita, percentage of health-service expenditure in the regional/provincial public affairs general budget, and ratio of urban to rural population, and the lowest percentage of illiteracy in females aged 15 and over. Northeast, south, and east China were high SES areas, while central, northwest, and southwest China were low SES areas. This classification accords with the pattern of economic growth and regional inequality in China during the reform era [25]. We found that cases living in low SES areas were more likely to be diagnosed at a later breast cancer stage than were those in high SES areas. 
Table 3 Individual pathologic characteristics by region

\begin{tabular}{|c|c|c|c|c|c|c|c|c|c|c|}
\hline & Total & $\begin{array}{l}\text { Beijing } \\
\text { (North) }\end{array}$ & $\begin{array}{l}\text { Liaoning } \\
\text { (Northeast) }\end{array}$ & $\begin{array}{l}\text { Hunan } \\
\text { (Central) }\end{array}$ & $\begin{array}{l}\text { Guangdong } \\
\text { (South) }\end{array}$ & $\begin{array}{l}\text { Zhejiang } \\
\text { (East) }\end{array}$ & $\begin{array}{c}\text { Shannxi } \\
\text { (Northwest) }\end{array}$ & $\begin{array}{c}\text { Sichuan } \\
\text { (Southwest) }\end{array}$ & $\chi^{2}$ & $\begin{array}{c}P- \\
\text { value }\end{array}$ \\
\hline \multirow[t]{2}{*}{$\begin{array}{l}\text { Pathological } \\
\text { Characteristics }\end{array}$} & $\begin{array}{c}\text { Total } N= \\
4211\end{array}$ & $\begin{array}{l}\text { Total } N= \\
\quad 641\end{array}$ & $\begin{array}{l}\text { Total } \mathrm{N}= \\
832\end{array}$ & $\begin{array}{l}\text { Total } N= \\
546\end{array}$ & $\begin{array}{l}\text { Total N = } \\
\quad 604\end{array}$ & $\begin{array}{l}\text { Total N }= \\
606\end{array}$ & $\begin{array}{c}\text { Total } N= \\
483\end{array}$ & $\begin{array}{c}\text { Total } \mathrm{N}= \\
499\end{array}$ & & \\
\hline & N (\%) & N (\%) & N (\%) & N (\%) & N (\%) & N (\%) & N (\%) & N (\%) & & \\
\hline \multicolumn{11}{|c|}{ Type of Postoperative Pathological Diagnosis ${ }^{(1)}$} \\
\hline $\begin{array}{l}\text { Carcinoma in Situ } \\
\text { (CIS) }\end{array}$ & $143(3.4)$ & $61(9.5)$ & $22(2.6)$ & $17(3.1)$ & $19(3.1)$ & $7(1.2)$ & $5(1.0)$ & $12(2.4)$ & 169.1 & $\begin{array}{c}< \\
0.001\end{array}$ \\
\hline $\begin{array}{l}\text { Invasive Ductal } \\
\text { Carcinoma }\end{array}$ & $3471(82.4)$ & $528(82.4)$ & $583(70.1)$ & $504(92.4)$ & $528(87.4)$ & $523(86.3)$ & $406(84.1)$ & $399(80.0)$ & & \\
\hline $\begin{array}{l}\text { Other Invasive } \\
\text { Carcinoma }\end{array}$ & $385(9.1)$ & $47(7.3)$ & $87(10.5)$ & $21(3.8)$ & $29(4.8)$ & $59(9.7)$ & $70(14.5)$ & $72(14.4)$ & & \\
\hline Other & $15(0.4)$ & $5(0.8)$ & $0(0.0)$ & $1(0.2)$ & $4(0.7)$ & $0(0.0)$ & $2(0.4)$ & $3(0.6)$ & & \\
\hline Unavailable & $197(4.7)$ & - & $140(16.8)$ & $3(0.5)$ & $24(4.0)$ & $17(2.8)$ & - & $13(2.6))$ & & \\
\hline \multicolumn{11}{|l|}{ Pathological stage ${ }^{(2)}$} \\
\hline Stage 0 & $39(0.9)$ & $7(1.1)$ & $13(1.6)$ & $6(1.1)$ & $8(1.3)$ & $4(0.7)$ & $0(0.0)$ & $1(0.2)$ & 450.5 & $\begin{array}{c}< \\
0.001\end{array}$ \\
\hline Stage I & $663(15.7)$ & $172(26.8)$ & $131(15.7)$ & 73 (13.4) & $98(16.2)$ & $95(15.7)$ & $77(15.9)$ & $17(3.4)$ & & \\
\hline Stage II & $1891(44.9)$ & $280(43.7)$ & $323(38.8)$ & $400(73.3)$ & $243(40.2)$ & $239(39.4)$ & $170(35.2)$ & $236(47.3)$ & & \\
\hline Stage III & $788(18.7)$ & $93(14.5)$ & $119(14.3)$ & $53(9.7)$ & $117(19.4)$ & $152(25.1)$ & $127(26.3)$ & $127(25.5)$ & & \\
\hline Stage IV & $113(2.7)$ & $2(0.3)$ & $4(0.5)$ & $3(0.5)$ & $20(3.4)$ & $5(0.8)$ & 56 (11.6) & $23(4.6)$ & & \\
\hline Unavailable & $717(17.1)$ & 87 (13.6) & $242(29.1)$ & $11(2.0)$ & $118(19.5)$ & $111(18.3)$ & $53(11.0)$ & $95(19.0)$ & & \\
\hline \multicolumn{11}{|l|}{ ER/PR test } \\
\hline Performed & $3555(84.4)$ & $616(96.1)$ & $711(85.5)$ & $535(98.0)$ & $511(84.6)$ & $462(76.2)$ & $282(58.4)$ & $438(87.8)$ & 483.2 & $\begin{array}{c}< \\
0.001\end{array}$ \\
\hline Not performed & $471(11.2)$ & $24(3.7)$ & $85(10.2)$ & $8(1.5)$ & $54(8.9)$ & $67(11.1)$ & $192(39.8)$ & $41(8.2)$ & & \\
\hline Unknown & $185(4.4)$ & $1(0.2)$ & $36(4.3)$ & $3(0.5)$ & $39(6.5)$ & $77(12.7)$ & $9(1.8)$ & $20(4.0)$ & & \\
\hline \multicolumn{11}{|l|}{ Her-2 test } \\
\hline Performed & $3251(77.2)$ & $609(95.0)$ & $693(83.3)$ & 533 (97.6) & $469(77.6)$ & $424(70.0)$ & $241(49.9)$ & $282(56.5)$ & 640.2 & $\begin{array}{c}< \\
0.001\end{array}$ \\
\hline Not performed & $762(18.1)$ & $31(4.8)$ & $98(11.8)$ & $8(1.5)$ & 99 (16.4) & $98(16.1)$ & $227(47.0)$ & $201(40.3)$ & & \\
\hline Unknown & $198(4.7)$ & $1(0.2)$ & $41(4.9)$ & $5(0.9)$ & $36(6.0)$ & $84(13.9)$ & $15(3.1)$ & $16(3.2)$ & & \\
\hline \multicolumn{11}{|l|}{ ER/PR status ${ }^{(3)}$} \\
\hline ER+\&PR+ & $1691(47.6)$ & $373(60.6)$ & $318(44.7)$ & $244(45.6)$ & $241(47.2)$ & $221(47.8)$ & $109(38.7)$ & $185(42.2)$ & 121.0 & $\begin{array}{c}< \\
0.001\end{array}$ \\
\hline$E R+\& P R-$ & $337(9.5)$ & $44(7.1)$ & 79 (11.1) & $54(10.1)$ & $37(7.2)$ & $41(8.9)$ & $31(11.0)$ & $51(11.6)$ & & \\
\hline ER-\&PR+ & $367(10.3)$ & 47 (7.6) & $108(15.2)$ & $32(6.0)$ & $75(14.7)$ & $34(7.4)$ & $37(13.1)$ & $34(7.8)$ & & \\
\hline ER-\&PR- & $1139(32.0)$ & $146(23.7)$ & $206(29.0)$ & $203(37.9)$ & $152(29.7)$ & $165(35.7)$ & $101(35.8)$ & $166(37.9)$ & & \\
\hline Unavailable & $21(0.6)$ & $6(1.0)$ & - & $2(0.4)$ & $6(1.2)$ & $1(0.2)$ & $4(1.4)$ & $2(0.5)$ & & \\
\hline \multicolumn{11}{|l|}{ Her-2 status ${ }^{(4)}$} \\
\hline Her-2 + & $736(22.6)$ & $286(47.0)$ & $36(5.2)$ & $156(29.3)$ & $90(19.2)$ & $68(16.0)$ & $29(12.0)$ & $71(25.2)$ & 339.6 & $\begin{array}{c}< \\
0.001\end{array}$ \\
\hline Her-2 - & $2113(65.0)$ & $299(49.1)$ & $545(78.6)$ & $314(58.9)$ & 337 (71.9) & $317(74.8)$ & $181(75.1)$ & $120(42.6)$ & & \\
\hline $\begin{array}{l}\text { Uncertain/ } \\
\text { unavailable }\end{array}$ & $402(12.4)$ & $24(3.9)$ & $112(16.2)$ & $63(11.8)$ & $42(9.1)$ & $39(9.2)$ & 31 (12.9) & $91(32.2)$ & & \\
\hline
\end{tabular}

(1): carcinoma in situ: ductal carcinoma in situ, lobular carcinoma in situ, microinvasive ductal carcinoma, and Paget's disease invasive ductal carcinoma: pure invasive ductal carcinoma and mixed invasive ductal carcinoma other invasive carcinomas: invasive labular carcinoma, tubular carcinoma, medullary carcinoma, mucin carcinoma, and other invasive carcinomas

(2): according to the AJCC/UICC TNM system

${ }^{(3)}$ : ER/PR status was based on all breast cancer cases tested for ER/PR

${ }^{(4)}$ : Her-2 status was based on all breast cancer cases tested for Her-2

Exactly how area-based socioeconomic conditions influence the stage at which an individual is diagnosed with breast cancer is complex. One explanatory factor is that individuals residing in areas with high GDP per capita are likely to have more personal income compared with those in low GDP per capita areas. In the United States, lack of ability to pay for screening services is often implicated as the reason why individuals 
Table 4 Incorporated SEls, area-based SES, and breast cancer stage at diagnosis

\begin{tabular}{|c|c|c|c|c|c|c|c|c|c|}
\hline \multirow[t]{2}{*}{$\begin{array}{l}\text { Variable by } \\
\text { level }\end{array}$} & \multirow[t]{2}{*}{ Mean \pm SD } & \multirow[t]{2}{*}{ Included Provinces } & \multirow[t]{2}{*}{ Total } & $\begin{array}{l}\text { Stage } \\
0 \& 1\end{array}$ & \multirow{2}{*}{$\begin{array}{l}\text { Stage II } \\
\text { N (\%) }\end{array}$} & \multirow{2}{*}{$\begin{array}{l}\text { Stage } \\
\text { III\&IV } \\
\mathrm{N}(\%)\end{array}$} & \multirow{2}{*}{$\begin{array}{c}\text { Unavailable } \\
\qquad \mathrm{N}(\%)\end{array}$} & \multirow[t]{2}{*}{$\begin{array}{l}\text { Chi-square for } \\
\text { trend }\end{array}$} & \multirow[t]{2}{*}{$\begin{array}{c}P- \\
\text { value }\end{array}$} \\
\hline & & & & N (\%) & & & & & \\
\hline \multicolumn{10}{|l|}{ GDP per capita } \\
\hline Highest & $\begin{array}{l}36146 \pm \\
17217\end{array}$ & Beijing & 641 & $\begin{array}{c}179 \\
(27.9)\end{array}$ & $\begin{array}{c}280 \\
(43.7)\end{array}$ & $95(14.8)$ & 87 (13.6) & 80.79 & $\begin{array}{c}< \\
0.001\end{array}$ \\
\hline High & $\begin{array}{l}20774 \pm \\
9006\end{array}$ & $\begin{array}{c}\text { Liaoning, Guangdong, } \\
\text { Zhejiang }\end{array}$ & 2024 & $\begin{array}{c}349 \\
(17.1)\end{array}$ & $\begin{array}{l}805 \\
(39.4)\end{array}$ & $417(20.4)$ & $471(23.1)$ & & \\
\hline Low & $8704 \pm 3986$ & Hunan, Shannxi, Sichuan & 1528 & $\begin{array}{c}174 \\
(11.4)\end{array}$ & $\begin{array}{c}806 \\
(52.7)\end{array}$ & $389(25.5)$ & $159(10.4)$ & & \\
\hline \multicolumn{10}{|c|}{ HSE percentage } \\
\hline Highest & $6.11 \pm 0.73$ & Beijing, Zhejiang & 1247 & $\begin{array}{l}278 \\
(22.3)\end{array}$ & $\begin{array}{c}519 \\
(41.6)\end{array}$ & $252(20.2)$ & $198(15.9)$ & 3.307 & 0.081 \\
\hline High & $4.42 \pm 0.57$ & Guangdong, Sichuan & 1103 & $\begin{array}{l}124 \\
(11.2)\end{array}$ & $\begin{array}{c}479 \\
(43.5)\end{array}$ & $287(26.0)$ & $213(19.3)$ & & \\
\hline Low & $3.49 \pm 0.70$ & Liaoning, Hunan, Shannxi & 1861 & $\begin{array}{c}300 \\
(16.1)\end{array}$ & $\begin{array}{c}893 \\
(48.0)\end{array}$ & $362(19.5)$ & $306(16.4)$ & & \\
\hline \multicolumn{10}{|l|}{ PU/PR ratio ${ }^{(3)}$} \\
\hline Highest & $5.39 \pm 0.22$ & Beijing & 641 & $\begin{array}{c}179 \\
(27.9)\end{array}$ & $\begin{array}{l}280 \\
(43.7)\end{array}$ & $95(14.8)$ & $87(13.6)$ & 72.00 & $\begin{array}{c}< \\
0.001\end{array}$ \\
\hline Higher & $1.67 \pm 0.09$ & Guangdong & 604 & $\begin{array}{c}106 \\
(17.5)\end{array}$ & $\begin{array}{c}243 \\
(40.2)\end{array}$ & $137(22.7)$ & 118 (19.6) & & \\
\hline High & $1.39 \pm 0.08$ & Liaoning, Zhejiang & 1438 & $\begin{array}{c}243 \\
(16.9)\end{array}$ & $\begin{array}{c}562 \\
(39.1)\end{array}$ & $280(19.5)$ & $353(24.5)$ & & \\
\hline Low & $0.62 \pm 0.08$ & Hunan, Shannxi, Sichuan & 1528 & $\begin{array}{c}174 \\
(11.4)\end{array}$ & $\begin{array}{l}806 \\
(52.7)\end{array}$ & $389(25.5)$ & $159(10.4)$ & & \\
\hline \multicolumn{10}{|c|}{ FPI percentage } \\
\hline Highest & $16.97 \pm 3.55$ & Zhejiang, Shannxi, Sichuan & 1588 & $\begin{array}{c}194 \\
(12.2)\end{array}$ & $\begin{array}{c}645 \\
(40.6)\end{array}$ & $490(30.9)$ & $259(16.3)$ & 135.83 & $\begin{array}{c}< \\
0.001\end{array}$ \\
\hline High & $10.60 \pm 2.62$ & Hunan, Guangdong & 1150 & $\begin{array}{c}185 \\
(16.1)\end{array}$ & $\begin{array}{c}643 \\
(55.9)\end{array}$ & $193(16.8)$ & $129(11.2)$ & & \\
\hline Low & $7.21 \pm 1.57$ & Beijing, Liaoning, & 1473 & $\begin{array}{c}323 \\
(21.9)\end{array}$ & $\begin{array}{c}603 \\
(40.9)\end{array}$ & $218(14.8)$ & $329(22.4)$ & & \\
\hline \multicolumn{10}{|c|}{$\underset{(5)}{\text { Area-based SES }}$} \\
\hline Highest & - & Beijing & 641 & $\begin{array}{c}179 \\
(27.9)\end{array}$ & $\begin{array}{c}280 \\
(43.7)\end{array}$ & $95(14.8)$ & 87 (13.6) & 80.79 & $\begin{array}{c}< \\
0.001\end{array}$ \\
\hline High & - & $\begin{array}{c}\text { Liaoning, Guangdong, } \\
\text { Zhejiang }\end{array}$ & 2024 & $\begin{array}{c}349 \\
(17.1)\end{array}$ & $\begin{array}{l}805 \\
(39.4)\end{array}$ & $417(20.4)$ & $471(23.1)$ & & \\
\hline Low & - & Hunan, Shannxi, Sichuan & 1528 & $\begin{array}{c}174 \\
(11.4)\end{array}$ & $\begin{array}{c}806 \\
(52.7)\end{array}$ & $389(25.5)$ & $159(10.4)$ & & \\
\hline
\end{tabular}

\footnotetext{
(1): GDP per capita: yuan per person per year (mean \pm SD, 1999-2008)
}

(2): percentage of health-services expenditure in the regional/province public affairs general budget (\%) (mean \pm SD, 1999-2008)

(3): ratio of urban to rural population (mean \pm SD, 2005-2008) (data is available in the National Bureau of Statistics of the People's Republic of China only for 2005 to 2008)

(4): percentage of illiteracy among women aged 15 and over (\%) (mean \pm SD, 1999-2008)

${ }^{(5)}$ : Area-based SES is a composite measure synthesized by cluster analysis of the four SEls

with low incomes, or those living in the poorest areas, have lower screening rates and are more likely to be diagnosed with cancer at a late stage [34]. In the present study, we observed that the patients from hospitals located in Beijing and Guangdong were diagnosed at an earlier stage than others. This may partly be explained by the routine breast cancer screening programs that have been implemented in these two cities for several years [32,33]. However, the screening programs in a city may not reflect the screening situation of the whole region. To evaluate the effect of routine screening on early diagnosis, a comprehensive prospective study is needed to compare districts with and without screening programs. 
Table 5 Multivariable logistic regression for association between individual demographic characteristics and breast cancer stage at diagnosis (stage 0 \& I, stage II, III \& IV), stratified by area-based SES*

\begin{tabular}{|c|c|c|c|c|}
\hline \multirow[t]{2}{*}{ Variable } & \multicolumn{2}{|c|}{ High SES areas } & \multicolumn{2}{|c|}{ Low SES areas } \\
\hline & OR $(95 \% \mathrm{Cl})$ & Wald $(P)$ & OR $(95 \% \mathrm{Cl})$ & Wald $(P)$ \\
\hline \multicolumn{5}{|l|}{ Age at Diagnosis (Years) } \\
\hline$\leq 40$ & 1.00 & & 1.00 & \\
\hline $40-69$ & $0.94(0.29-3.09)$ & $0.01(0.92)$ & $1.18(0.43-3.23)$ & $0.10(0.75)$ \\
\hline$\geq 70$ & $1.02(0.70-1.51)$ & $0.02(0.90)$ & $1.40(0.85-2.29)$ & $1.75(0.19)$ \\
\hline \multicolumn{5}{|l|}{ Body Mass Index $\left(\mathrm{kg} / \mathrm{m}^{2}\right)$} \\
\hline$\leq 24.99$ & 1.00 & & 1.00 & \\
\hline$\geq 25.00$ & $1.37(0.92-2.06)$ & $2.39(0.12)$ & $1.09(0.40-12.97)$ & $0.03(0.86)$ \\
\hline \multicolumn{5}{|l|}{ Occupation } \\
\hline Homemaker & 1.00 & & 1.00 & \\
\hline Manual worker & $0.81(0.33-2.02)$ & $0.20(0.65)$ & $0.18(0.05-0.64)$ & $6.99(0.01)$ \\
\hline White-collar worker & $0.77(0.33-1.80)$ & $0.37(0.54)$ & $0.26(0.08-0.88)$ & $4.66(0.03)$ \\
\hline Other & $1.21(0.45-3.25)$ & $0.14(0.70)$ & $0.18(0.05-0.65)$ & $6.85(0.01)$ \\
\hline \multicolumn{5}{|l|}{ Education } \\
\hline University and above & 1.00 & & 1.00 & \\
\hline High school & $2.89(1.30-6.44)$ & $6.76(0.01)$ & $0.42(0.07-2.36)$ & $0.97(0.32)$ \\
\hline Middle school & $2.77(132-5.79)$ & $7.27(0.01)$ & $0.97(0.42-2.21)$ & $0.01(0.94)$ \\
\hline Primary school & $1.87(0.99-3.64)$ & $3.43(0.06)$ & $0.98(0.47-2.05)$ & $0.01(0.96)$ \\
\hline None & $1.64(0.87-3.08)$ & $2.34(0.13)$ & $0.74(0.40-1.36)$ & $0.93(0.34)$ \\
\hline \multicolumn{5}{|l|}{ Marital Status } \\
\hline Married & 1.00 & & 1.00 & \\
\hline Single & $6.27(0.81-48.76)$ & $3.08(0.08)$ & $1.01(0.11-9.51)$ & $0.001(0.99)$ \\
\hline Widowed/Divorced & $1.18(0.14-10.06)$ & $0.02(0.88)$ & $0.49(0.15-1.62)$ & $1.38(0.24)$ \\
\hline \multicolumn{5}{|l|}{ ER/PR status } \\
\hline $\mathrm{ER}+\& \mathrm{PR}+$ & 1.00 & & 1.00 & \\
\hline ER+\&PR- & $0.97(0.50-1.90)$ & $0.01(0.93)$ & $1.10(0.54-2.23)$ & $0.07(0.79)$ \\
\hline ER-\&PR+ & $1.57(0.83-2.95)$ & $1.96(0.16)$ & $0.73(0.36-1.51)$ & $0.70(0.40)$ \\
\hline ER-\&PR- & $1.04(0.69-1.56)$ & $0.04(0.85)$ & $1.31(0.81-2.11)$ & $1.19(0.28)$ \\
\hline
\end{tabular}

*: Education information for $50.3 \%$ of cases in the highest SES area was unknown, so we did not analyze the effect of individual demographic characteristics in this subgroup

Local governments with a high percentage of healthservice expenditure in their regional/provincial public affairs general budgets (HSE percentage) may provide better medical services, giving residents more access to health care such as breast cancer screening, diagnosis, and treatment, which in turn results in earlier diagnosis. Barrya and Breen found that residence in economically and socially distressed or medically underserved neighborhoods tends to increase the likelihood of late-stage cancer diagnoses [19]. However, we observed no clear trend association between HSE percentage and breast cancer stage at diagnosis. HSE percentage may not be a direct indicator of the level of medical service of an area, since HSE percentage may be inconsistent with an area's economic development. For instance, while northeast China had high GDP per capita, it was the lowest HSE percentage of the areas studied.

In contrast, we found an association between earlier breast cancer stage at diagnosis and higher ratio of urban to rural population (PU/PR ratio). In China, there is wide SES gap between cities and rural areas, and so urban/rural status is highly correlated with SES. A higher PU/PR ratio hints that more individuals in the area live in cities, and thus may have more access to medical services and ability to afford medical insurance than do residents of rural areas. This would in turn make them more likely to seek prompt medical care for any health problems. These differences may contribute to disparities in breast cancer stage at diagnosis. Several studies have shown that patients with health insurance are less likely to be diagnosed at a late stage of breast cancer $[22,35]$, an effect similar to that found for other cancers, such as colorectal cancer $[34,36]$.

In this study, area-based education was measured as percentage of illiteracy in females aged 15 and over (FPI percentage), emphasizing education of females. We found a trend association between FPI percentage and stage at diagnosis: the higher the FPI percentage in an area, the later the stage of breast cancer at which women in the area were diagnosed. After stratified 
analysis by area-based SES, individual education level appeared to influence the stage at diagnosis in high SES areas, while in low SES areas, working women were less likely to be diagnosed at late breast cancer stages than were homemakers. These results highlight the importance of education in high SES areas. Education may be a proxy variable representing individual knowledge and behaviors toward prevention and screening of breast cancer. Women with lower education level may also have higher risk of alcohol-related death and diseases [37]. Low education itself may also create barriers to receiving recommended screening, since low health literacy, low general literacy, and language barriers impact an individual's ability to navigate the medical service system, understand screening options and recommendations, and communicate with healthcare professionals [38]. Working women in low SES areas were more likely to be diagnosed at early stages, which may result from their high breast cancer screening attendance relative to that of homemakers. The results from our previous study [39] and a study by Damiani et al. [40] show that education and occupation were positively associated with breast cancer screening attendance, which in turn may influence breast cancer stage at diagnosis.

However, after adjusting for area-based SES, we found no significant associations between other individual demographic characteristics and breast cancer stage at diagnosis. Thus, area-based SES appeared to be the main, underlying factor influencing breast cancer stage at diagnosis. Baquet et al. similarly found that SES predicted the likelihood of a group's access to education, certain occupations, and health insurance, as well as income level and living conditions, all of which are associated with a person's chances of being diagnosed with late stage disease and of surviving cancer [41]. Moreover, Singh et al. [42] and Launay et al. [43] found that at every stage of diagnosis, breast cancer patients from lower-income areas had lower 5-year relative survival rates did than did those from higher-income areas. The presence of additional illnesses and treatment disparities may contribute to these differences $[44,45]$. Compared with less developed areas, more developed areas possess better detection techniques, and their cases have the ability to pay more for more accurate tests and more effective therapy. For instance, we found that in Beijing, the highest SES area, more than 95\% of cases were tested for ER/PR/Her-2. In most of the low SES areas, however, only about half of cases were tested for Her-2. Even so, this pattern was in agreement with the guidelines of the Breast Health Global Initiative (BHGI), which contend that Her-2 measurement is problematic in limited-resource settings due to the high cost of immunohistochemical analysis, fluorescence in situ hybridization, and trastuzumab therapy, and recommend introducing this test only at the maximal-resource level [46].

The present study is the first geographically epidemiologic study of breast cancer in China. Although we only included women with breast cancer attended to these 7 regional referral hospitals, we expect the effect of SES and later stage of diagnosis will be much stronger than we found in this study (if we included those women attended at local hospitals, such county hospitals). Our findings thus provide a baseline for understanding the country's patient and tumor characteristics. Our evaluation of associations between breast cancer stage and area-based SES, as well as individual demographic characteristics stratified by areabased SES, may help to identify high risk areas and the most important and controllable regional and individual factors influencing breast cancer stage at diagnosis. However, this was an ecological study, so we must recognize the possibility of ecological fallacy. In addition to the individual-level variables examined in this study, it would be useful to examine the effects of other variables, such as access to breast cancer screening services, alcohol use, family history of breast cancer, and lack of exercise, as they may affect breast cancer stage at diagnosis by influencing women's attitudes and behaviour to breast cancer prevention and screening. However, their effects could not be analyzed in this study due to the high proportion of missing data; we plan to test the effects of these variables on breast cancer stage in a future study. Another limitation is that we lacked data on individual SES, which was likely strongly correlated with both education level and area-based SES. Although our method of calculating area-based SES may not have assessed actual socioeconomic conditions with complete accuracy, our results for area-based SES accord with the pattern of economic growth and regional inequality in China during the reform era [25]. Thus, we think this indicator has some validity. However, a more comprehensive, validated area-based SES measurement is needed that takes into consideration factors such as concentration of poverty, health insurance coverage, proportion of the population with blue collar jobs, unemployment rate, median household income, and median value of owner-occupied houses [47]. Finally, area-based SES was measured at a large geographic scale in our study; it would be instructive to study area-based SES differences among smaller geographic areas in a future study.

\section{Conclusion}

In summary, the effects of area-based SES are complex and multidimensional. Women in lower SES areas may be more likely to ignore symptoms for a variety of 
economic, social, and cultural reasons. Additionally, women in lower SES areas tend to be less educated and may not understand disease progression, and so may not seek treatment until breast cancer has developed into a late stage. WHO's national cancer control programmes suggest that research to determine the most cost-effective cancer control strategies is especially relevant in developing countries, perhaps even more so than in industrialized countries. However, the available research data for effective and efficient cancer control decision-making are extremely limited in these countries. Our results suggest that in China and other developing countries with similar socioeconomic conditions, breast cancer control programs should focus on ensuring adequate access to screening and improving breast cancer awareness and education. This would facilitate diagnosis at an early stage, particularly for populations living in socioeconomically disadvantaged areas, and would in turn decrease breast cancer mortality and improve surviving patients' quality of life.

\section{Abbreviations}

SES: Area-based socioeconomic status; SEls: Socioeconomic indicators; SEER: Surveillance Epidemiology and End Results; HSE: percentage Percentage of health-service expenditure in the regional/provincial public affairs general budget; PU/PR: ratio Ratio of urban to rural population; FPI: percentage Percentage of illiteracy in females aged 15 and over; CICAMS: Chinese Academy of Medical Sciences; AJCC: American Joint Committee on Cancer; ER: Estrogen receptor; PR: Progestin receptor; Her-2: Human epidermal growth factor receptor 2; CRF: Case report form; CBE: Clinical breast examination; MRI: Magnetic resonance imaging; BHGI: Breast Health Global Initiative

\section{Acknowledgements}

We thank the Cancer Institute of the Chinese Academy of Medical Sciences (CICAMS) with providing their expertise in the development of the study. We also thank the local investigators from Beijing, Liaoning (Shenyang), Hunan (Changsha), Guangdong (Guangzhou), Zhejiang (Hangzhou), Shannxi (Xi'an), and Sichuan (Chengdu) for data collection and assisting us complete the project successfully. The authors also thank Pfizer for funding the research and Shawna Williams for editing this text.

\section{Author details}

'Department of Epidemiology, West China School of Public Health, Sichuan University, 16 Ren Min Nan Lu, Chengdu, Sichuan 610041, China. ${ }^{2}$ Department of Cancer Epidemiology, Cancer Institute \& Hospital, Chinese Academy of Medical Sciences \& Peking Union Medical College, 17 South Panjiayuan Lane, Beijing 100021, China. ${ }^{3}$ Department of Pathology, Cancer Institute \& Hospital, Chinese Academy of Medical Sciences \& Peking Union Medical College, 17 South Panjiayuan Lane, Beijing 100021, China. ${ }^{4}$ Center of Breast Disease, Cancer Institute \& Hospital, Chinese Academy of Medical Sciences \& Peking Union Medical College, 17 South Panjiayuan Lane, Beijing 100021, China. ${ }^{5}$ Department of Breast Surgery, Liaoning Cancer Hospital, 44 Xiaoyanhe Road, Dadong District, Shenyang 110042, China. ${ }^{6}$ Department of Breast Surgery, Zhejiang Cancer Hospital, 38 Banshanqiao Guanji Road, Hangzhou 310022, China. ${ }^{7}$ Department of Breast Oncology, Sun Yat-Sen University Cancer Center, 651 Dongfeng East, Gungzhou 510060, China. ${ }^{8}$ Department of Breast-thyroid Surgery, Xiangya Sencod Hospital, Central South University, 139 Renminzhonglu, Changsha 410011, China. ${ }^{9}$ Department of Breast Surgery, the Second People's Hospital of Sichuan Province, Chengdu 610041, China. ${ }^{10}$ Department of Oncosurgery, the First Affiliated Hospital of Medical College, Xi'an Jiao Tong University, 277 Yanta West Road, Xi'an 710061, China.

\section{Authors' contributions}

QW helped to analyze, and interpret the data. She also drafted the initial manuscript. JL helped to design the study and interpret the data. SZ helped design the CRF to collect the data. JYL was the local PI who helped with data collection and performed critical revisions of the manuscript. JHF, YP, and RH helped with the data management and analysis. BNZ helped to design the study and is the clinical PI of the study. BZ, HJY, XMX, ZHT, HL, and JJH were the local PIs who helped with data collection. YLQ was the PI of this study and assisted in designing the study and performed critical revisions of the manuscript. All authors read and approved the final manuscript.

\section{Competing interests}

The authors declare that they have no competing interests.

Received: 20 November 2011 Accepted: 29 March 2012

Published: 29 March 2012

\section{References}

1. IARC: Cancer Epidemiology Database, GLOBOCAN. 2008 [http://www-dep. iarc.fr].

2. Horner MJ, Ries LAG, Krapcho M, et al: SEER Cancer Statistics Review. National Cancer Institute. Bethesda, MD; 2006 [http://seer.cancer.gov/csr/ 1975_2006/].

3. Parkin DM, Whelan SI, Ferlay J, Raymond L, Young J: Cancer Incidence in Five Continents Lyon: IARC Scientific Publications; 1997.

4. Parkin DM, Whelan SI, Ferlay J, Teppo L, Thomas DB: Cancer Incidence in Five Continents Lyon: IARC Scientific Publications; 2002.

5. Bray F, McCarron P, Parkin DM: The changing global patterns of female breast cancer incidence and mortality. Breast Cancer Res 2004, 6:229-239.

6. Curado MP, Edwards B, Shin HR, Storm H, Ferlay J, Heanue M, Boyle P. Cancer Incidence in Five Continents Lyon: IARC Scientific Publications; 2007.

7. IARC: Cancer Epidemiology Database, GLOBOCAN. 2002 [http://www-dep. iarc.fr/].

8. Tan SM, Evans AJ, Lam TP, Cheung KL: How relevant is breast cancer screening in the Asia/Pacific region? The Breast 2007, 16:113-119.

9. Sadler GR, Wang K, Wang M, Ko CM: Chinese women: behaviors and attitudes towards breast cancer education and screening. Women Health Issues 2000, 10(1):20-26.

10. Breen N, Wagener DK, Brown ML, Davis WW, Ballard-Barbash R: Progress in cancer screening over a decade: Results of cancer screening from the 1987, 1992, and 1998. National Health Interview Surveys 2001, 93(22):1704-1713.

11. Diez Roux AV: Investigating neighborhood and area effects on health American Journal of Public Health 2001, 91(11):1783-1789.

12. Kawachi I, Berkman LF: Neighborhoods and Health Oxford: Oxford University Press; 2003.

13. Krieger N, Chen JT, Waterman PD, Rehkopf DH, Subramanian SV: Painting a truer picture of US socioeconomic and racial/ethnic health inequalities: the Public Health Disparities Geocoding Project. American Journal of Public Health 2005, 95(2):312-323.

14. Singh G, Miller BA, Hankey BF, Edwards BK: NCl Cancer Surveillance Monograph Series Bethesda, MD: National Cancer Institute; 2003.

15. Imlach Gunasekara F, Carter KN, Liu I, Richardson K, Blakely T: The relationship between income and health using longitudinal data from New Zealand. Journal of Epidemiol and Community Health 2011, [Epub ahead of print].

16. Shepherd CC, Li J, Zubrick SR: Socioeconomic disparities in physical health among Aboriginal and Torres Strait Islander children in Western Australia. Ethn Health 2012, [Epub ahead of print].

17. Audureau E, Rican S, Coste J: Worsening trends and increasing disparities in health-related quality of life: evidence from two French populationbased cross-sectional surveys, 1995-2003. Qual Life Res 2012, [Epub ahead of print].

18. Yu XQ: Socioeconomic disparities in breast cancer survival: relation to stage at diagnosis, treatment and race. BMC Cancer 2009, 9:364.

19. Barry J, Breen $\mathrm{N}$ : The importance of place of residence in predicting latestage diagnosis of breast or cervical cancer. Health \& Place 2005, 11:15-29.

20. Merkin SS, Stevenson L, Powe N: Geographic socioeconomic status, race, and advanced-stage breast cancer in New York City. American Journal of Public Health 2002, 92:64-70. 
21. Schwartz KL, Crossley-May H, Vigneau FD, Brown K, Banerjee M: Race, socioeconomic status and stage at diagnosis for five common malignancies. Cancer Causes \& Control 2003, 14:761-766.

22. MacKinnon JA, Duncan RC, Huang Y, Lee DJ, Fleming LE, Voti L, Rudolph M, Wilkinson JD: Detecting an association between socioeconomic status and late stage breast cancer using spatial analysis and area-based measures. Cancer Epidemiology Biomarkers \& Prevention 2007, 16:756-762.

23. Roche LM, Skinner R, Weinstein RB: Use of a geographic information system to identify and characterize areas with high proportions of distant stage breast cancer. Journal of Public Health Management \& Practice 2002, 8:26-32.

24. Sheehan TJ, DeChello LM: A space-time analysis of the proportion of late stage breast cancer in Massachusetts, 1988-1997. International Journal of Health Geographics 2005, 4:15.

25. $X u$ ZY, Li ST: Analysis on the trend of regional income disparity in China. Economic Research Journal 2006, 7:106-116, in Chinese.

26. Li J, Zhang BN, Fan JH, et al: A Nation-wide Multicenter 10-year (19992008) Retrospective Clinical Epidemiological Study of Breast Cancer in China. BMC Cancer 2011, 11(1):364.

27. Fleming ID, Cooper JS, Henson DE, et al: AJCC Cancer Staging Manual. 5 edition. Philadelphia: Lippincott Raven; 1997

28. Greene F, Page D, Fleming I, Fritz A, Balch C, Haller D, Morrow M: AJCC Cancer Staging Manual. 6 edition. Philadelphia: Springer; 2002.

29. Diez Roux AV: The study of group-level factors in epidemiology: rethinking variables, study designs, and analytical approaches. Epidemiologia \& Prevenzione 2004, 26:104-111.

30. Pickett KE, Pearl M: Multilevel analyses of neighbourhood socioeconomic context and health outcomes: a critical review. Journal of Epidemiol and Community Health 2001, 55(2):111-122.

31. US Census Bureau: US Department of Commerce Economics and Statistics Administration. Washington, DC; 2005 [http://www2.census.gov/ census_2000/datasets/Summary_File_3/New_Jersey/].

32. Liu L, Ding $H$, Wang LY, et al: Analysis on the General Survey Result of Gynecological Diseases in Beijing Area from 2000 to 2005. Chinese General Practice 2006, 15:1275-1276, in Chinese.

33. Wu QL, Zeng FL, Zeng XQ, Li Ping, Su HM: The analysis of breast cance screening in Guangzhou from 1994 to2003. Chinese Primary Health Care 2004, 18(10):52-54.

34. Henry KA, Sherman R, Roche LM: Colorectal cancer stage at diagnosis and area socioeconomic characteristics in NewJersey. Health \&Place 2009, 15:505-513.

35. Halpern MT, Bian J, Ward EM, Schrag NM, Chen AY: Insurance status and stage of cancer at diagnosis among women with breast cancer. Cancer 2007, 110:403-411.

36. Matthews BA, Anderson RC, Nattinger AB: Colorectal cancer screening behavior and health insurance status (United States). Cancer Causes \& Control 2005, 16:735-742

37. WHO: Global status report on alcohol and health. 2011 [http://www.who. int/substance_abuse/publications].

38. James TM, Greiner KA, Ellerbeck EF, Feng C, Ahluwalia JS: Disparities in colorectal cancer screening: a guideline-based analysis of adherence. Ethnicity \& Disease 2006, 16:228-233.

39. Huang $Y$, Zhou K, Li H, et al: Screening among women from different socio-economic regions in southwest China: A cross-sectional study. Asian Pacific J Cancer Prev 2011, 12:203-209.

40. Damiani G, Federico B, Basso D, Ronconi A, Bianchi CB, Anzellotti GM, Nasi G, Sassi F, Ricciardi W: Socioeconomic disparities in the uptake of breast and cervical cancer screening in Italy: a cross sectional study. BMC Public Health 2012, [Epub ahead of print].

41. Baquet $C R$, Commiskey P: Socioeconomic factors and breast carcinoma in multicultural women. Cancer 2000, 88:1256-1264.

42. Singh GK, Miller BA, Hankey BF, Edwards BK: Area socioeconomic variation in US cancer incidence, mortality, stage, treatment, and survival 1975-1999 Bethesda, MD, National Cancer Institute; 2003, NCI Cancer Surveillance Monograph Series, Number 4.

43. Launay L, Dejardin O, Pornet C, Morlais F, Guittet L, Launoy G, Bouvier V: Influence of socioeconomic environment on survival in patients diagnosed with esophageal cancer: a population-based study. Dis Esophagus 2012, [Epub ahead of print].

44. Joslyn SA: Racial differences in treatment and survival from early-stage breast carcinoma. Cancer 2002, 95(8):1759-1766.
45. Smedley B, Stith A, Nelson A: Unequal treatment: confronting racial and ethnic disparities in health care, Committee on Understanding and Eliminating Racial and Ethnic Disparities in Health Care Institute of Medicine, Washington, DC: National Academy Press; 2002.

46. Shyyan R, Masood S, Badwe RA, Errico KM, Liberman L, Ozmen V, Stalsberg H, Vargas H, Vass L: Global Summit Diagnosis and Pathology Panel: Breast cancer in limited-resource countries: diagnosis and pathology. Breast Journal 2006, 12(Suppl 1):S27-37.

47. Yin D, Morris C, Allen M, Cress R, Bates J, Liu L: Does socioeconomic disparity in cancer incidence vary across racial/ethnic groups? Cancer Causes \& Control 2010, 21(10):1721-30

\section{Pre-publication history}

The pre-publication history for this paper can be accessed here: http://www.biomedcentral.com/1471-2407/12/122/prepub

doi:10.1186/1471-2407-12-122

Cite this article as: Wang et al:: Breast cancer stage at diagnosis and area-based socioeconomic status: a multicenter 10-year retrospective clinical epidemiological study in China. BMC Cancer 2012 12:122.

\section{Submit your next manuscript to BioMed Central and take full advantage of:}

- Convenient online submission

- Thorough peer review

- No space constraints or color figure charges

- Immediate publication on acceptance

- Inclusion in PubMed, CAS, Scopus and Google Scholar

- Research which is freely available for redistribution

Submit your manuscript at www.biomedcentral.com/submit
C Biomed Central 